\title{
Desigualdades no pré-natal em cidade do Sudeste do Brasil
}

\section{Inequalities in prenatal care in a southeastern city in Brazil}

Sandra Costa Fonseca ${ }^{1}$

Daniela da Silva Alves Monteiro ${ }^{2}$

Camila Moraes de Souza Camacho Pereira ${ }^{3}$

Ana Carolina Daflon Scoralick ${ }^{3}$

Mariana Gomes Jorge ${ }^{1}$

Suelem do Rozario ${ }^{4}$

${ }^{1}$ Departamento de

Epidemiologia e

Bioestatística, Instituto de

Saúde da Comunidade,

Universidade Federal

Fluminense. R. Marquês de

Paraná $303 / 3^{\circ}$ andar/sala 6 ,

Centro. 24.030-215 Niterói

RJ Brasil.

sandracfonseca@yahoo.com.br

${ }^{2}$ Hospital Universitário

Antônio Pedro, UFF.

${ }^{3}$ Prefeitura Municipal

Angra dos Reis.

${ }^{4}$ Instituto Nacional do

Câncer.
Abstract The scope of this article is to evaluate the association between adequate prenatal care and sociodemographic variables in Niterói in the state of Rio de Janeiro. It involved a cross-sectional study conducted between 2000 and 2009 evaluating 62,449 live births using data from the Brazilian Live Birth Information System (SINASC). Multivariate analysis by logistic regression was performed considering quantitatively adequate prenatal care - seven or more visits - as the dependent variable, and maternal age, educational level and skin color as independent variables. The time trends of mother's age, educational level and prenatal visits were also analyzed. The significance level was 5\%. There was an improvement in educational level, reduction in adolescent pregnancy and an increase in mothers aged over 35 in Niterói. Women who attended seven or more prenatal visits remained above $80 \%$, though with differences according to age, education and skin color. Adult women (OR = 1.4; IC95\% 1.39-1.56). women with eight or more years of schooling (OR $=2.5 ;$ IC95\% 2.45-2.70) and white women $(O R=$ 2.4; IC95\% 2.30-2.53) had more chances of adequate prenatal care. Health inequalities in maternal health care on offer in Niterói were identified, despite improvements in social and demographic indicators in the city.

Key words Prenatal care, Health inequalities, Adolescence, Education, Time series
Resumo $O$ artigo tem por objetivo avaliar a associação de variáveis demográficas e sociais com o pré-natal adequado na cidade de Niterói, RJ. Estudo transversal, realizado de 2000 a 2009. Foram estudados 62.449 nascidos vivos, por meio do Sistema de Informação sobre Nascidos Vivos (SINASC). A análise multivariada por regressão logística considerou como variável dependente o pré-natal quantitativamente adequado - sete ou mais consultas - e como variáveis independentes: idade, escolaridade materna, cor da pele. Também foi analisada a tendência temporal de idade e escolaridade maternas, número de consultas de pré-natal. O nível de significância foi 5\%. Houve aumento da escolaridade, redução de gravidez na adolescência e aumento de mães com mais de 35 anos em Niterói. O percentual de mulheres com sete ou mais consultas se manteve acima de 80\%, com diferenças de acordo com idade, escolaridade e cor da pele. Tiveram mais chances de pré-natal adequado: adultas (OR=1,4; IC95\% 1,39-1,56); com oito anos ou mais de estudo $(O R=2,5 ;$ IC95\% 2,45-2,70); e brancas (OR=2,4; IC95\% 2,30-2,53). Identificamos desigualdades na atenção à saúde materna oferecida em Niterói, apesar da melhora temporal dos indicadores sociais e demográficos na cidade.

Palavras-chave Cuidado pré-natal, Desigualdade em saúde, Adolescente, Escolaridade, Série temporal 


\section{Introdução}

A atenção pré-natal de qualidade e humanizada é fundamental para a saúde materna e neona$\mathrm{tal}^{1,2}$, por incluir ações de prevenção e promoção da saúde da gestante e do feto, identificando situações de risco para ambos e permitindo intervenções oportunas ${ }^{3}$.

A cobertura do pré-natal vem aumentando no Brasil $1^{4}$; contudo, os estudos sobre a qualidade do atendimento mostram que ainda há muito que avançar ${ }^{5}$. Embora haja restrições aos estudos apenas quantitativos do pré-natal, existe evidência da associação entre o número de consultas e os desfechos perinatais ${ }^{6}$.

A despeito da alta cobertura do pré-natal do Brasil, persistem desigualdades na atenção oferecida. Em Belo Horizonte, Friche et al. ${ }^{7}$ realizaram estudo ecológico em 2001 e identificaram concentração dos piores indicadores materno-infantis nas regiões com baixo nível socioeconômico: comparecimento a menos de quatro consultas pré-natais, mães com menos de oito anos de estudo e maior número de mulheres adolescentes.

Outros estudos mostram que para mães adolescentes, mais que a vulnerabilidade etária, as condições de pobreza e a baixa escolaridade contribuem para menor acesso ao pré-natal ${ }^{8,9}$.

O estudo de Miranda et al..$^{10}$, realizado em Vitória (ES), identificou fatores associados com número de consultas em maternidades públicas no ano de 2007. Neste cenário, apenas $67,1 \%$ assistiram a seis ou mais consultas. Ter mais de oito anos de escolaridade e rendimento de mais de 600 dólares por mês aumentaram a chance de número adequado de consultas entre estas mulheres $^{10}$. Em Campinas, SP, entre 2001 e 2002, a utilização dos serviços de saúde também foi desigual. As gestantes de menor renda iniciaram o pré-natal mais tardiamente e fizeram um número menor de consultas ${ }^{3}$. Na cidade de Rio Grande, no sul do país, observou-se em 2007 que a renda familiar foi diretamente proporcional ao número de consultas de pré-natal e à qualidade de atendimento ${ }^{11}$. Na mesma cidade, observouse que no setor privado o número de gestantes que iniciou o pré-natal no primeiro trimestre, o número de consultas de pré-natal e a realização de exames clínicos de rotina foram maiores ${ }^{12}$. Em Aracaju (SE), em 2005, a assistência pré-natal inadequada se relacionou, entre outros fatores, à baixa escolaridade e à baixa renda familiar ${ }^{13}$.

Além da renda, diferenças raciais também são observadas, como no Rio de Janeiro, RJ, no período de 1999 a 2001. Houve uma persistente situa- ção desfavorável das mulheres de pele preta e parda em relação às brancas, quanto ao pré-natal adequado ${ }^{14}$. Em Pelotas (RS), em 2004, houve forte associação entre etnia e renda, estando mulheres negras principalmente no grupo de menor renda e menor número de consultas de pré-natal ${ }^{15}$. Já em Porto Alegre, RS, no ano de 2002, Bassani et al. mostraram que um maior rendimento reduz os riscos de um pré-natal inadequado; enquanto cor da pele "não branca", após ajuste para variáveis socioeconômicas, não mostrou associação ${ }^{16}$.

Vale ressaltar que tanto o estudo de Aracaju ${ }^{13}$ como o de Porto Alegre ${ }^{16}$, analisaram o número de gestações ou de filhos vivos anteriores e mostraram que as mulheres com mais filhos - provavelmente por condições sociais - também têm maior dificuldade de frequentar o pré-natal.

A maioria destes estudos usou dados primários, exceto Friche et al. ${ }^{7}$, que trabalharam com o Sistema de informações sobre nascidos vivos (SINASC). O SINASC vem sendo progressivamente utilizado na pesquisa em saúde materna e infantil, e, paralelamente, estudos apontam que a qualidade das informações do sistema está aumentando nos últimos anos ${ }^{17,18}$.

Em Niterói (RJ), apenas um estudo foi realizado em 1992, usando informações do SINASC na cidade e discutindo os possíveis fatores de risco à saúde infantil. Este estudo destacou o elevado número de cesarianas realizado na cidade e a relação inversa entre baixo peso ao nascer e escolaridade materna. Não foram descritos aspectos do pré-natal ${ }^{19}$.

Justifica-se descrever aspectos quantitativos da atenção pré-natal na cidade e identificar desigualdades na sua utilização, com a finalidade de subsidiar o planejamento de ações de saúde materno-infantil no município.

\section{Métodos}

Niterói integra a região metropolitana II do estado do Rio de Janeiro. Conta com uma população estimada de 487.562 habitantes (2010) sendo a sexta cidade mais populosa do estado e a de maior Índice de Desenvolvimento Humano, com $100 \%$ da população residindo em área urbana ${ }^{20}$.

Os serviços de saúde do Município de Niterói encontram-se territorialmente organizados em seis regionais de saúde, contando, para atenção básica, com Unidades de Médico da Família, Unidades Básicas de Saúde e Policlínicas Comunitárias $^{21}$. As estatísticas vitais são processadas pelo 
Observatório de Saúde da Fundação Municipal de Saúde.

A fonte de dados deste estudo foi o SINASC, sendo usados os bancos de dados cedidos pela Fundação Municipal de Saúde de Niterói.

Foram analisadas as seguintes variáveis: idade materna ( $\leq 19$ anos, 20-34 anos, 35 e mais); grau de escolaridade da mãe (baixa: $<8$ anos de estudo; intermediária: 8-11 anos, alta: $\geq 12$ anos de estudo), número de consultas de pré-natal (menor que três, quatro a seis, sete ou mais consultas), história reprodutiva (número de gestações, número de nascidos vivos e mortos), tipo de gravidez (única, múltipla), tipo de parto (vaginal, cesáreo), raça (branca, preta e parda), número de filhos vivos ( $\leq 1$ e 2 ou mais). Para as variáveis maternas e assistenciais, as mulheres com partos múltiplos contaram apenas uma vez na totalização dos dados. A forma de categorização das variáveis obedeceu à disponibilidade das informações do SINASC. Por isto, adotamos o ponto de corte de sete ou mais consultas como ideal em termos quantitativos, já que a categoria de 4 a 6 consultas não permite avaliar o número exato. Deve ser ressaltado que na nova estratégia Rede Cegonha, do Ministério da Saúde, o número de sete consultas será o ponto de corte adotado como indicador do pré-natal ${ }^{22}$.

As variáveis foram avaliadas segundo a tendência temporal, para o período de 2000 a 2009. A análise de tendência foi feita por meio de regressão, usando o software Joinpoint Regression Program $^{23}$. Essa análise ajusta uma série de linhas e seus pontos de junção, em uma escala log, testando as tendências anuais. $\mathrm{O}$ teste de significância é o Monte Carlo Permutation method (que ajusta a melhor linha para cada segmento). Uma vez que estes segmentos são estabelecidos, o percentual anual de mudança (APC) é estimado e testado. Quando há um ponto de junção em que o sentido se inverte, os períodos são analisados separadamente.

Para avaliar possíveis desigualdades na utilização do pré-natal, utilizou-se o desenho transversal, considerando todo o período de 2000 a 2009. O desfecho analisado foi o pré-natal adequado e as variáveis independentes foram: idade, escolaridade materna, cor da pele do recémnascido (como proxy para cor materna), ajustadas também para número de filhos vivos.

Foi analisado o pré-natal adequado do ponto de vista quantitativo - sete ou mais consultas, com adaptação para as mulheres com idade gestacional menor que 37 semanas, como sugerido por outros autores ${ }^{3,16}$. De acordo com a ca- tegorização da variável no SINASC, consideramos como número adequado de consultas: para aquelas entre 32 e 36 semanas, cinco; entre 28 e 31 semanas, quatro; entre 22 e 27 semanas, três; e pelo menos duas consultas para as mulheres com menos de 22 semanas. Como não havia informação sobre início do pré-natal na declaração e nascido vivo no período estudado foi usado apenas o critério de número de consultas.

Para a análise multivariada, as variáveis independentes foram dicotomizadas. Os dados do banco do SINASC foram processados e analisados por meio do aplicativo Statistical Package for the Social Sciences (SPSS) versão 17.0. A análise multivariada foi realizada por regressão logística, sendo estimadas as odds ratios ajustadas e seus respectivos intervalos de confiança (95\%).

Este estudo foi aprovado pelo Comitê de Ética em pesquisa do HUAP, obedecendo à Resolução CNS no 196/96 $6^{24}$, não implicando qualquer risco para as puérperas e seus bebês. Os dados foram coletados do banco de declarações de nascido vivo (DN) de Niterói de forma confidencial.

\section{Resultados}

Foram incluídos no estudo 62.449 nascimentos vivos no período de 2000 a 2009. A incompletitude da informação foi baixa para a maioria das variáveis. Idade e escolaridade da mãe tiveram menos que $0,1 \%$ de ausência. Idade gestacional e pré-natal apresentaram $0,5 \%$ e $1 \%$ de incompletitude, respectivamente. A informação sobre cor da pele estava ausente em 1,9\%.

Observou-se uma redução anual constante de mães adolescentes $(2,3 \%)$ e aumento da gravidez em mulheres com mais de 35 anos (2,9\%), ambos estatisticamente significantes. Houve melhoria no nível educacional, com uma redução anual (5\%) do percentual com baixa escolaridade (Tabela 1).

A proporção de mulheres com menos de três consultas de pré-natal reduziu de 5,8\% (2000) para $3,7 \%$ (2009). Na análise de tendência, essa redução não apresentou significância estatística. Quanto ao percentual de mulheres com sete ou mais consultas, manteve-se constante, em torno de $81 \%$, porém com diferenças de acordo com idade, escolaridade e raça/cor da pele (Tabela 1).

Entre as adolescentes, o percentual médio, de 2000 a 2009, de mulheres com pré-natal adequado foi menor que o das outras faixas etárias ( $\mathrm{Ta}$ bela 2). Considerando a tendência (Figura 1), observou-se que a partir de 2002 o grupo de ado- 


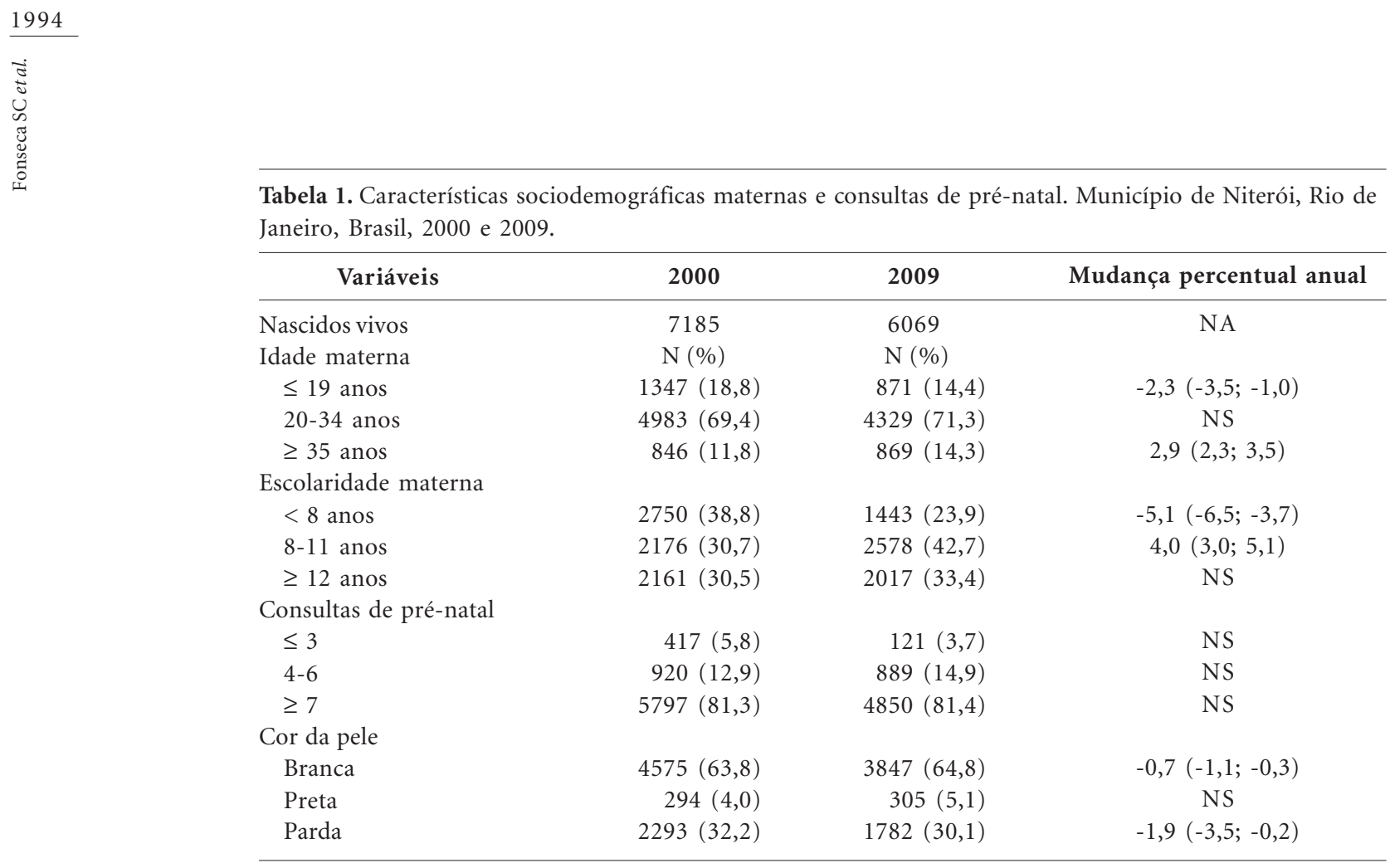

Tabela 2. Pré-natal adequado, de acordo com variáveis sociodemográficas. Município de Niterói, Rio de Janeiro, Brasil, 2000 a 2009.

\begin{tabular}{lcc}
\hline \multicolumn{1}{c}{ Variáveis } & $\begin{array}{c}\text { Percentual de mulheres com número adequado } \\
\text { de consultas para idade gestacional }\end{array}$ & p-valor (tendência) \\
\hline Idade materna & $74,5 \%$ & $\mathrm{p}=0,00$ \\
$\leq 19$ anos & $85,8 \%$ & \\
$20-34$ anos & $90,6 \%$ & \\
$\geq 35$ anos & $71,4 \%$ & $\mathrm{p}=0,00$ \\
Escolaridade materna & $85,0 \%$ & \\
$<8$ anos & $96,7 \%$ & $\mathrm{p}=0,00$ \\
$8-11$ anos & & \\
$\geq 12$ anos & $68,8 \%$ & \\
Cor da pele & $74,8 \%$ & \\
Preta & $90,8 \%$ & \\
Parda & & \\
Branca & & \\
\hline
\end{tabular}

lescentes vem apresentando queda deste percentual de forma constante e estatisticamente significativa (2,25\% anualmente). Para as mais velhas, a situação é inversa; continua aumentando o percentual de mulheres que realizam sete ou mais consultas (0,5\% ao ano). Na faixa de $20-34$ anos não se detectaram mudanças.

Dentre as mulheres com menos de oito anos de estudo, 71,4\% conseguiram realizar pré-natal adequado, comparadas a $85 \%$ daquelas com 8 a 11 anos e $96,7 \%$ das com 12 ou mais anos, ob- servando-se gradiente nestes percentuais, de acordo com a escolaridade (Tabela 2).

$\mathrm{Na}$ análise de tendência temporal, para as mulheres com escolaridade baixa e intermediária (Figura 2), a continuidade do pré-natal vem piorando, com diminuição constante e significativa de $0,77 \%$ e $0,97 \%$ ao ano (a partir de 2003 para esta faixa de escolaridade), respectivamente. As mulheres com escolaridade alta, que já têm um percentual elevado, não tiveram mudança no período. 

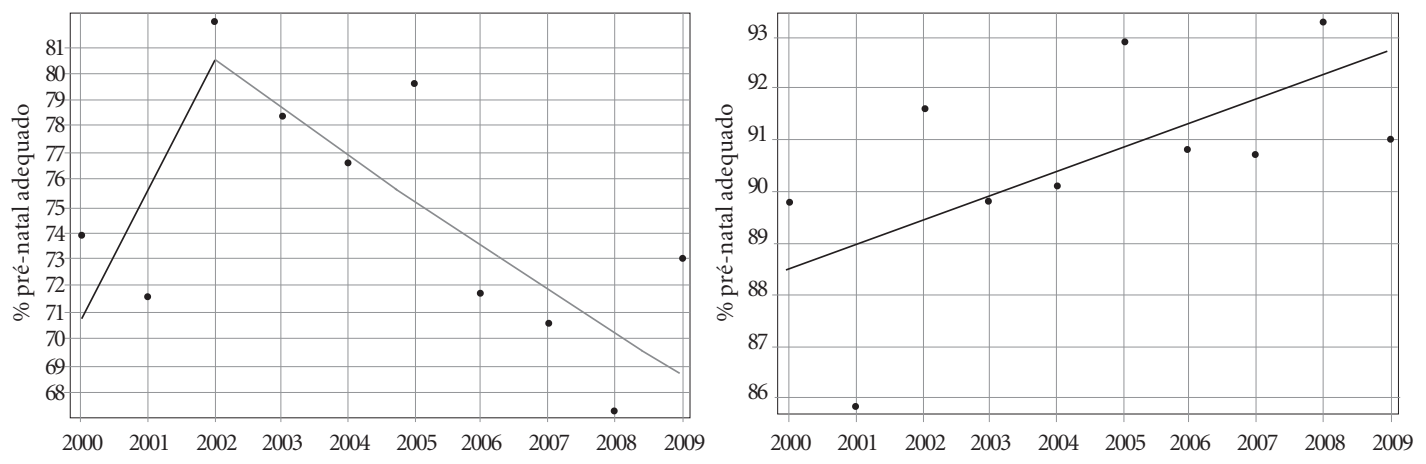

$$
\begin{array}{ll}
\text { adolescentes } & \text { Observed } \\
\text { - APC } 1=6.63 \\
\text { - APC } 2=-2.25^{*}
\end{array}
$$

$$
35 \text { anos ou mais } \begin{aligned}
& - \text { Observed } \\
& -\mathrm{APC}=0.52^{*}
\end{aligned}
$$

Figura 1. Série temporal do percentual de mulheres com pré-natal adequado, de acordo com idade materna: adolescentes e mulheres com 35 ou mais anos.

* $\mathrm{p}<0,05$. APC - mudança percentual anual.

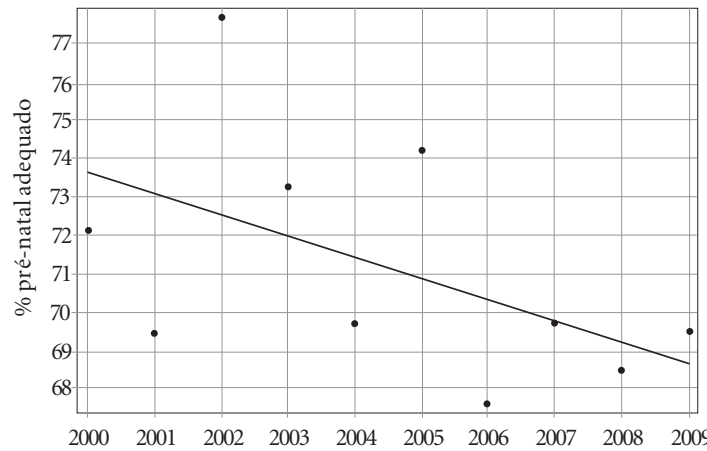

$2000 \quad 2001 \quad 2002 \quad 2003 \quad 2004 \quad 2005 \quad 2006 \quad 2007 \quad 2008 \quad 2009$

$$
\begin{array}{l|l}
\text { escolaridade baixa } & \begin{array}{l}
- \text { Observed } \\
-\mathrm{APC}=-0.77^{*}
\end{array}
\end{array}
$$

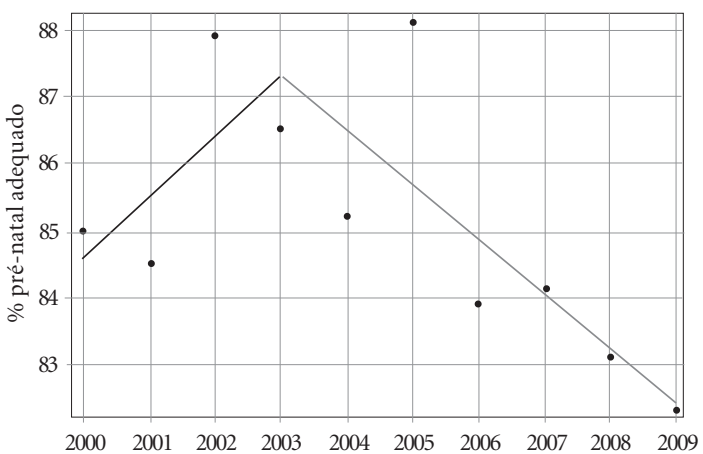

$$
\text { escolaridade intermediária } \begin{aligned}
& - \text { Observed } \\
& \text { - APC } 1=1.07 \\
& - \text { APC } 2=-0.50^{*}
\end{aligned}
$$

Figura 2. Série temporal do percentual de mulheres com pré-natal adequado, de acordo com escolaridade materna: baixa e intermediária. ${ }^{\star} \mathrm{p}<0,05$. APC - mudança percentual anual.

Considerando a variável cor da pele, apenas $68,8 \%$ das mulheres de cor preta conseguiram o pré-natal adequado, enquanto as pardas obtive- ram $74,8 \%$ e as brancas atingiram 90,8\% (Tabela 2). Não se observaram tendências para esta variável no período de 2000 a 2009. 
$\mathrm{Na}$ análise multivariada, considerando também o número de filhos vivos, cada um destes fatores mostrou-se associado com o desfecho. Como mostrado na Tabela 3, tiveram mais chances de fazer um pré-natal adequado: mulheres adultas $(\mathrm{OR}=1,87)$; mulheres com oito anos ou mais de estudo (OR = 2,02; IC95\% 2,45-2,70), e mulheres brancas $(\mathrm{OR}=2,19$; IC95\% 2,30-2,53).

\section{Discussão}

O presente estudo investigou a associação de variáveis demográficas e sociais - idade, escolaridade e cor da pele -, com pré-natal adequado em Niterói, RJ. Estas variáveis tiveram ótima completitude no SINASC da cidade, permitindo a análise quantitativa e de tendência temporal.

Em relação à idade materna, Niterói vem mudando o perfil etário das gestantes, com diminuição da gravidez em adolescentes e aumento em mulheres com mais de 35 anos. A frequência de gravidez na adolescência tem mostrado decréscimo em todo o país ${ }^{25}$, mas a intensidade da queda em Niterói se mostrou maior que no Brasil. Para as adolescentes de Niterói que ainda vivenciam a gravidez, persiste uma desvantagem em relação às mulheres mais velhas, com menor frequência de pré-natal adequado, ressaltando-se que esta desigualdade vem se agravando ano a ano.

Quanto ao grau de escolaridade, a cidade tem incrementado o nível educacional da população e consegue manter melhor cobertura de pré-natal $(71,4 \%)$, para mulheres com menos de oito anos

Tabela 3. Associação das variáveis sociodemográficas com pré-natal adequado. Município de Niterói, Rio de Janeiro, Brasil, 2000 a 2009. Análise multivariada por regressão logística.

\begin{tabular}{lcc}
\hline \multicolumn{1}{c}{ Variáveis } & $\begin{array}{c}\text { Odds ratio } \\
\text { ajustadas }\end{array}$ & p-valor \\
\hline $\begin{array}{l}\text { Idade materna } \\
\quad \text { Adultas }\end{array}$ & $1,87(1,77-1,98)$ & 0,000 \\
$\quad$ Adolescentes & 1,00 & \\
Escolaridade materna & & \\
$\quad \geq 8$ anos & $2,02(2,45-2,70)$ & 0,000 \\
$\quad<8$ anos & 1,00 & \\
Cor da pele & & \\
$\quad$ Branca & $2,19(2,09-2,30)$ & 0,000 \\
$\quad$ Não Branca & 1,00 & \\
Número de filhos vivos & & \\
$\quad \leq 1$ & $1,85(1,75-1,96)$ & 0,000 \\
$>1$ & 1,00 & \\
\hline
\end{tabular}

de estudo, em relação a Belo Horizonte ${ }^{7}$, que apresentou uma média de $42,4 \%$ e de Vitória ${ }^{10}$, com $46 \%$ de cobertura. Ainda assim, é preocupante que mulheres mais vulneráveis, por sua baixa escolaridade, tenham em Niterói um menor percentual de pré-natal adequado, comparadas àquelas com escolaridade alta e que esta diferença venha aumentando. Este resultado também foi observado em Vitória ${ }^{10}$ e $\mathrm{Aracaju}^{13}$, onde o aumento da escolaridade da mãe representou um menor risco de pré-natal inadequado. No entanto, há resultados discordantes na literatura, como em Porto Alegre ${ }^{16}$, onde, após o ajuste para renda, a escolaridade não se mostrou associada.

Quanto à cor da pele, Niterói apresentou desempenho semelhante a Pelotas ${ }^{15}$, onde houve associação entre etnia e qualidade da assistência pré-natal: em ambas, mulheres da raça negra possuíram um menor número de consultas de pré-natal. Esta associação não é consensual na literatura. No caso de Porto Alegre, após ajustes de variáveis socioeconômicas, não se observou a associação entre etnia "não branca" e pré-natal inadequado.

Portanto, mulheres com maior vulnerabilidade para riscos maternos e perinatais são as que têm menor frequência de cuidado pré-natal, ratificando o conceito de "cuidado inverso"26, onde a disponibilidade da assistência varia inversamente com as necessidades da população servida.

Como limitações do presente estudo, temos a impossibilidade de ajuste para variáveis não presentes no SINASC, como renda, que tem se mostrado associada ao desfecho do pré-natal e que pode confundir ou modificar o efeito das variáveis aqui investigadas. Outra limitação se refere à qualidade dos dados do SINASC ${ }^{27}$. Apesar da elevada completitude, não há estudos sobre a confiabilidade das variáveis do SINASC no município de Niterói. No município do Rio de Janeiro foi identificado excelente desempenho para idade materna (Intervalo de Correlação Intraclasse = $0,981)$ e desempenho bom a moderado para escolaridade (kappa $=0,66$ ) e consultas de pré-natal (kappa $=0,62)^{28}$. Segundo Romero e Cunha ${ }^{17}$, comparado aos dados do Censo, a tendência de erro na variável escolaridade é para os níveis mais elevados - o SINASC costuma registrar percentuais maiores -, enquanto para baixa escolaridade, a concordância é alta. Para cor da pele, o SINASC costuma registrar $6 \%$ a mais de brancos e o correspondente em menor número de pardos. A idade materna tem excelente consistência. Por último, o caráter transversal do estudo não permite assunções de causalidade. 
A despeito destas limitações, acreditamos que ficaram evidenciadas iniquidades na atenção prénatal na cidade de Niterói e que a exploração dos dados de forma temporal se mostrou uma ferramenta útil para subsidiar o monitoramento de indicadores de saúde.

Deve ser ressaltado que as pacientes com maior vulnerabilidade, seja pela baixa escolaridade ou pela idade reprodutiva precoce, são atendidas preferencialmente na rede pública ${ }^{29} \mathrm{e}$ que, portanto, estes resultados são mais aplicáveis no contexto da saúde pública.

\section{Colaboradores}

SC Fonseca foi responsável pela concepção e delineamento do artigo e supervisão de todas as etapas; DSA Monteiro, CMMSC Pereira e ACD Scoralick contribuíram na revisão bibliográfica, interpretação dos dados e na redação do artigo; MG Jorge e S Rozario contribuíram revisão bibliográfica, na análise e interpretação dos dados e redação do artigo. Todos os autores realizaram revisão crítica da versão do artigo encaminhada à revista.

\section{Agradecimentos}

Ao CNPq pela bolsa de Mestrado de Mariana Gomes Jorge e Suelem do Rozario durante a realização deste trabalho.

\section{Conclusão}

Apesar de Niterói vir apresentando melhora temporal dos indicadores sociais e demográficos, identificamos desigualdades na atenção à saúde materna oferecida na cidade, mensurada pelo número de consultas de pré-natal. Devem ser realizados esforços no sentido de ampliar o acesso e garantir a continuidade do pré-natal, principalmente para as mulheres em situação de vulnerabilidade.

\section{Referências}

1. Brasil. Ministério da Saúde (MS). Secretaria de Políticas de Saúde. Programa de Humanização do Pré-natal e Nascimento. Brasília: MS; 2000.

2. Serruya SJ, Lago TG, Cecatti JG. O panorama da atenção pré-natal no Brasil e o Programa de Humanização do Pré-natal e Nascimento. Rev Bras Saúde Matern Infant 2004; 4(3):269-279.

3. Almeida SDM, Barros MBA. Equidade e atenção à saúde da gestante em Campinas (SP), Brasil. Rev Panam Salud Publica 2005; 17(1):15-25.

4. Victora CG, Aquino EM, Leal MC, Monteiro CA, Barros FC, Szwarcwald CL. Maternal and child health in Brazil: progress and challenges. Lancet 2011; 377(9780):1863-1876.

5. Andreucci CB, Cecatti JG. Desempenho de indicadores de processo do Programa de Humanização do Pré-natal e Nascimento no Brasil: uma revisão sistemática. Cad Saude Publica 2011; 27(6):1053-1064.

6. Dowswell T, Carroli G, Duley L, Gates S, Gülmezoglu AM, Khan-Neelofur D, Piaggio GG. Alternative versus standard packages of antenatal care for lowrisk pregnancy. Cochrane Database Syst Rev 2010; (10):CD000934.

7. Friche AA, Caiaffa WT, César CC, Goulart LM, Almeida MC. Indicadores de saúde materno infantil em Belo Horizonte, Minas Gerais, Brasil, 2001: análise dos diferenciais intraurbanos. Cad Saude Publica 2006; 22(9):1955-1965.

8. Cesar JA, Mendoza-Sassi RA, Gonzalez-Chica DA Mano PS, Goulart-Filha SM. Características sociodemográficas e de assistência à gestação e ao parto no extremo sul do Brasil. Cad Saude Publica 2011; 27(5):985-994. 
9. Gama SG, Szwarcwald CL, Sabroza AR, Castelo Branco V, Leal MC. Fatores associados à assistência pré-natal precária em uma amostra de puérperas adolescentes em maternidades do Município do Rio de Janeiro, 1999-2000. Cad Saude Publica 2004; 20 (Supl. 1):S101-S111.

10. Miranda AE, Trindade CR, Nunes RH, Marba EF, Fernandes MC, Quarto GH, França LC. Factors associated with prenatal care and seeking assistance in public hospitals in Vitória, Espírito Santo, Brazil. Women Health 2010; 50(3):229-240.

11. Gonçalves CV, Cesar JA, Mendoza-Sassi RA. Qualidade e equidade na assistência à gestante: um estudo de base populacional no Sul do Brasil. Cad Saude Publica 2009; 25(11):2507-2516.

12. Cesar JA, Mano PS, Carlotto K, Gonzalez-Chica DA, Mendoza-Sassi RA. Público versus privado: avaliando a assistência à gestação e ao parto no extremo sul do Brasil. Rev Bras Saúde Matern Infant 2011; 11(3):257-263.

13. Ribeiro ER, Guimarães AM, Bettiol $\mathrm{H}$, Lima DD, Almeida ML, de Souza L, Silva AA, Gurgel RQ. Risk factors for inadequate prenatal care use in the metropolitan area of Aracaju, Northeast Brazil. BMC Pregnancy Childbirth 2009; 9:31.

14. Leal MC, Gama SG, Cunha CB. Desigualdades raciais, sociodemográficas e na assistência ao prénatal e ao parto, 1999-2001. Rev Saude Publica 2005; 39(1):100-107.

15. Victora CG, Matijasevich A, Silveira MF, Santos IS, Barros AJD, Barros FC. Socio-economic and ethnic group inequities in antenatal care quality in the public and private sector in Brazil. Health Policy Plan 2010; 25(4):253-261.

16. Bassani DG, Surkan PJ, Olinto MT. Inadequate use of prenatal services among Brazilian women: the role of maternal characteristics. Int Perspect Sex Reprod Health 2009; 35(1):15-20.

17. Romero ED, Cunha CB. Avaliação da qualidade das variáveis epidemiológicas e demográficas do Sistema de Informações sobre Nascidos Vivos, 2002. Cad Saude Publica 2007; 23(3):701-714.

18. Paiva NS, Coeli CM, Moreno AB, Guimaraes RM, Camargo KR. Sistema de informações sobre nascidos vivos: um estudo de revisão. Cien Saude Colet 2011; 16(Supl. 1):1211-1220.

19. Kale PL. Primeira Avaliação do Sistema de Informações sobre Nascidos Vivos no Município de Niterói. Cad Saude Coletiva 1997; 5(1):53-64.
20. Instituto Brasileiro de Geografia e Estatística (IBGE). Cidades. [página na Internet] [acessado 2012 nov 10]. Disponível em: http://www.ibge.gov.br/cidadesat/ painel/painel.php?codmun=330330\#.

21. DATASUS. Cadastro Nacional de Estabelecimentos de Saúde. [página na Internet] [acessado 2012 nov 5] Disponível em: http://cnes.datasus.gov.br/Listar Mantidas.asp?VCnpj=32556060000181.

22. Brasil. Ministério da saúde (MS). Diretrizes gerais e operacionais da Rede Cegonha. [acessado 2013 jul 15]. Disponível em: http://portal.saude.gov.br/portal/ saude/gestor/visualizar_texto.cfm?idtxt $=37082$

23. National Cancer Institute. Joinpoint Regression Program, Version 3 - April 2011; Statistical Methodology and Applications Branch and Data Modeling Branch, Surveillance Research Program National Cancer Institute. [acessado 2012 jul 2]. Disponível em: http://surveillance.cancer.gov/joinpoint/

24. Brasil. Ministério da Saúde (MS). Conselho Nacional de Saúde. Resolução no ${ }^{\circ} 196$ de 10 de outubro de 1996. Diretrizes e Normas Regulamentadoras de Pesquisas Envolvendo Seres Humanos. Diário Oficial da União 1996; 16 out.

25. Pinto e Silva JL, Surita FGC. Gravidez na adolescência: situação atual. Rev Bras Ginecol Obstet 2012; 34(8):347-350.

26. Hart JT. The inverse care law. Lancet 1971; 1(7696): 405-412.

27. Pedraza DF. Qualidade do Sistema de Informações sobre Nascidos Vivos (Sinasc): análise crítica da literatura. Cien Saude Colet 2012; 17(10):2729-2737.

28. Theme Filha MM, Gama SGN, Cunha CB, Leal MC. Confiabilidade do Sistema de Informações sobre Nascidos vivos Hospitalares no município do Rio de Janeiro, 1999-2001. Cad Saude Publica 2004; 20(Supl. 1):S83-S91.

29. Zimmermmann JB, Nani ACG, Junqueira CB, Iani GCM, Bahia GGS. Aspectos ginecológicos e obstétricos de pacientes atendidas nos serviços público e privado de saúde. Há diferenças? Rev Bras Ginecol Obstet 2011; 33(12):401-407.

Artigo apresentado em 15/04/2013

Aprovado em 12/07/2013

Versão final apresentada em 18/07/2013 